\title{
Genetic and pathologic characteristics of infectious bursal disease viruses isolated from broiler chickens in Iran during 2014-2015
}

\author{
H. NOROUZIAN ${ }^{1}$, G. FARJANIKISH ${ }^{2}$, H. HOSSEINI ${ }^{3}$
}

\begin{abstract}
${ }^{1}$ Department of Clinical Sciences, School of Veterinary Medicine, Lorestan University, P. O. Box: 465, Khorramabad, Iran; ${ }^{2}$ Department of Pathobiology, School of Veterinary Medicine, Lorestan University, Khorramabad, Iran; ${ }^{3}$ Department of Clinical Sciences, Faculty of Veterinary Medicine, Islamic Azad University, Karaj, Iran
\end{abstract}

\begin{abstract}
Summary. - Infectious bursal disease (IBD) virus causes a highly contagious immunosuppressive disease in chickens. A total number of 12 pooled bursal samples were collected during 2014-2015 from broiler farms in different regions of Iran. Typical macroscopical and histopathological lesions of the bursa of Fabricius were found similar to reports by other researchers. A 474-bp part of hypervariable region of VP2 (hvVP2) was sequenced and analyzed. Ten isolates had the characteristic amino acid residues of very virulent IBD (vvIBD) viruses and the other two were identified as attenuated (vaccine) strains. The vvIBD isolates had a unique $G$ to $S$ mutation at position 254, compared to other Iranian vvIBD isolates. Two attenuated isolates had the mutation 253Q, not found in D78 strain, creating virulent variant of vaccine strains. Degree of similarity among the studied vvIBD isolates was relatively high (97.6-100\%), proposing a common ancestor for them. However, they were partly different from previous Iranian and neighbor countries' isolates (96.2-97.3\% similarity to Shiraz isolate and $95.7-96.7 \%$ to Iraq and Turkey isolates). In phylogenetic analysis, the studied vvIBD isolates classified as a separate subgroup in the group of isolates from Iran and neighbor countries. More studies on genetic and antigenic characteristics of these isolates as well as probable modifications in their pathogenicity are needed to evaluate the significance of the mentioned differences.
\end{abstract}

Keywords: very virulent IBD; histopathology; VP2; phylogenetic analysis

\section{Introduction}

Infectious bursal disease (IBD), traditionally named as Gumboro disease, is an important immunosuppressive disease of chickens, depleting premature B lymphocytes in the bursa of Fabricius (Lasher and Shane, 1994). Although there is a long history of the disease since its first discovery in the USA in 1962 (Cosgrove, 1962), IBD is currently widespread in poultry farms all over the world, also in Iran. The first report of IBD in Iran dates back to 1981 (Aghakhan et al., 1996).

IBD virus is a non-enveloped, double-stranded RNA virus, classified based on virulence into classical virulent, very virulent (vv) and subclinical variant (van den Berg

E-mail: noroozianh@yahoo.com; phone: +989161596032 (mobile). Abbreviations: hvVP2 = highly variable viral protein 2; IBD = infectious bursal disease; $\mathrm{vv}=$ very virulent et al., 2004). Very virulent form of IBD was first seen in Europe in 1989 (Van den Berg et al., 1991), causing up to $70 \%$ mortality in chicken flocks. Cases of vvIBD have been previously reported in Middle East countries, like Iraq (Amin and Jackwood, 2014), Turkey (Ceribaci et al., 2014), Egypt (Mohamed et al., 2014), and Iran (Hosseini, 2004).

The large segment of IBD virus genome (A) encodes four structural viral proteins: VP2, VP4, VP3 and VP5 (Becht, 1981). VP2 has many serotype-specific antigenic sites responsible for induction of protective immunity against IBD virus (Snyder et al., 1988). The variable domain of VP2 segment is changed by a high rate of mutation, especially in the hypervariable region (hvVP2), altering amino acid residues responsible for antigenic variation. The hvVP2 region has major and minor hydrophilic peaks, in which amino acid residue changes may result in antigenic diversities (Vakharia et al., 1994). The region has been used for pathotypes differentiation and phylogenetic 
analysis of IBD viruses (Jackwood, 2004). Hence, it is not surprising that scientists around the world have been focusing on molecular characterization of VP2 gene, particularly its hypervariable region (Bayliss et al., 1990; Amin and Jackwood, 2014; Jenberie et al., 2014).

On the other hand, pathotype of vvIBD is not only concerned with the sequence of hv VP2 region, but also with the VP1 gene in segment B (Le Nouën et al., 2006). Therefore, a complete understanding of molecular basis of pathogenicity of IBD virus needs the identification of this part of the virus genome.

So far, different kinds of vaccines have been widely used to control IBD outbreaks in poultry farms of Iran. Nevertheless, outbreaks of IBD have occurred in vaccinated flocks, particularly in recent years. One probable reason is that strains with new antigens can escape the protection induced by commercially available vaccines (Jackwood et al., 2001). Interestingly, most of recent vvIBD cases in different regions of Iran have not resulted in a high mortality rate, which is typical of vvIBD outbreaks in the first years of its emergence (Hosseini, 2004). Our aim was to investigate the up to date situation of vvIBD in broiler farms of Iran, based on molecular characteristics of the hvVP2 region.

\section{Materials and Methods}

Necropsy and histopathology. A total number of 12 pooled bursal samples were collected during 2014-2015 from broiler and layer farms in different regions of Iran. At necropsy, tissue specimens were collected and fixed in $10 \%$ neutral buffered formalin for histopathological studies. Fixed tissue samples were further processed, embedded in paraffin, sectioned, stained with hematoxylin and eosin (H\&E) and then studied microscopically.

$R N A$ extraction and RT-PCR. The virus RNA was extracted using RNeasy Mini Kit (Qiagen, Germany), according to the manufacturer's instructions. Reverse Transcription (RT) was performed with RevertAid First Strand cDNA Synthesis Kit (Thermo scientific, Canada). For PCR, primers B3 (5'-CCCAGAGTCTACACCATA-3') and B4 (5'-TCCTGTTGCCACTCTTTC-3') were used to amplify the hypervariable sequence in hvVP2 (474-bp region) (Lin et al., 1993). The PCR was conducted at $95^{\circ} \mathrm{C}$ for $2 \mathrm{~min}$, followed by 35 cycles at $95^{\circ} \mathrm{C}$ for $1 \mathrm{~min}, 53^{\circ} \mathrm{C}$ for $90 \mathrm{~s}, 72^{\circ} \mathrm{C}$ for $1 \mathrm{~min}$. At the end of amplification cycles, a final extension at $72^{\circ} \mathrm{C}$ for $7 \mathrm{~min}$ was done. The reaction mixture was composed of $5 \mu \mathrm{l}$ of cDNA, $2 \mu \mathrm{l}$ of forward and reverse primers (each $20 \mathrm{pmol}$ ), and $25 \mu \mathrm{l}$ of PCR master mix (CinnaGen, Iran). PCR products were subjected to electrophoresis in a $1 \%(\mathrm{w} / \mathrm{v})$ agarose gel and DNA fragments of the expected length were extracted and purified with the GeneJET Gel Extraction Kit (Thermo scientific, Canada). Purified samples were sent for nucleotide sequencing (Bioneer, South Korea). The acquired Acc. Nos. of sequences from GenBank were: KU532133 to KU532143, KX017536 and KX017537.
Sequence and phylogenetic analysis. Nucleotide and deduced amino acid sequences were edited with the EditSeq program, version 5 (DNASTAR Inc., USA). Pairwise sequence alignments were performed with the Clustal W alignment algorithm using the MegAlign program, version 5 (DNASTAR Inc.), on the obtained sequences and some other hvVP2 sequences of IBD isolates available in GenBank. Sequence similarities and phylogenetic relationships of different isolates were also studied with the MegAlign program.

\section{Results}

\section{Necropsy and histopathological analysis}

Studied chicken flocks suffered from depression, diarrhea, ruffled feather, etc. In gross pathology, variable degrees of nephritis, hepatomegaly, bursal hemorrhage and, particularly when the disease proceeded, airsacculitis, pericarditis, perihepatitis and bursal atrophy were seen. Mortality rate in all farms was less than 20\% (Table 1).

Histopathological analysis of the bursa revealed degeneration and necrosis of B lymphocytes in medullary region of bursal follicles, presence of depleted follicles, depleted lymphocytes were replaced by heterophils and debris cells. Histopathological changes in some of the affected bursa of Fabricius mainly showed distended interfollicular spaces with inflammatory cell infiltration, hyperemia and mild to moderate follicular atrophy. Presence of cysts in the follicles was also evident. Sections of spleen revealed lymphocyte depletion in splenic lymphoid follicles and heterophils invasion. Degeneration of hepatocytes in the livers and tubular cell necrosis in the kidneys were also observed (Fig. 1).

\section{Sequence analysis}

A total number of 10 studied isolates were classified as vvIBD virus according to their hvVP2 sequences, and two other isolates were classified as attenuated (vaccine) strains (Table 1). Isolates IR/H1396.1/15, IR/H1396.2/15, IR/ H1396.4/15, IR/H1396.5/15 and IR/H1396.6/15 were identical to each other ( $100 \%$ of similarity), but were relatively different from isolates IR/H1446.7/15 and IR/H1446.9/15 (97.6-97.8\% of similarity). The similarity between two attenuated isolates was $96.1 \%$. Nucleotide similarity between the vvIBD isolates and attenuated isolates ranged from $89.1 \%$ to $92.4 \%$. Degree of similarity between studied Iranian vvIBD isolates and vvIBD isolates from other countries was from 95.2 (BJ2012.1 from China) to 96.9\% (UPM9204 from Malaya).

In comparison of the deduced amino acid sequences of studied vvIBD isolates, there was a similarity between 98 and $100 \%$. The well-known markers of the very virulent pathotype, including amino acid residues A222, I242, I256, 
Table 1. Characteristics of studied IBD isolates

\begin{tabular}{llccc}
\hline Virus isolate & $\begin{array}{l}\text { Amino acid } \\
\text { characteristics }\end{array}$ & Type of bird & $\begin{array}{c}\text { Mortality rate of } \\
\text { flock (\%) }\end{array}$ & $\begin{array}{c}\text { Geographic } \\
\text { location }\end{array}$ \\
\hline IR/H1396.1/15 & Very virulent & Broiler & 9 & Lorestan \\
IR/H1396.2/15 & Very virulent & Broiler & 8 & Ilam \\
IR/H1396.4/15 & Very virulent & Broiler & 12 & Golestan \\
IR/H1396.5/15 & Very virulent & Broiler & 18 & Lorestan \\
IR/H1396.6/15 & Very virulent & Broiler & 8 & Tehran \\
IR/H1446.6/15 & Very virulent & Broiler & 9 & Qazvin \\
IR/H1446.7/15 & Very virulent & Broiler & 10 & Qazvin \\
IR/H1446.9/15 & Very virulent & Broiler & 12 & Khorasan \\
IR/H1548.1/16 & Very virulent & Layer & 6 & KU532137 \\
IR/H1548.1/16 & Very virulent & Layer & 5 & KU532139 \\
IR/H1446.2/15 & Attenuated & Broiler & 10 & Alborz \\
IR/H1446.10/15 & Attenuated & Broiler & 12 & Isfahan \\
\hline
\end{tabular}
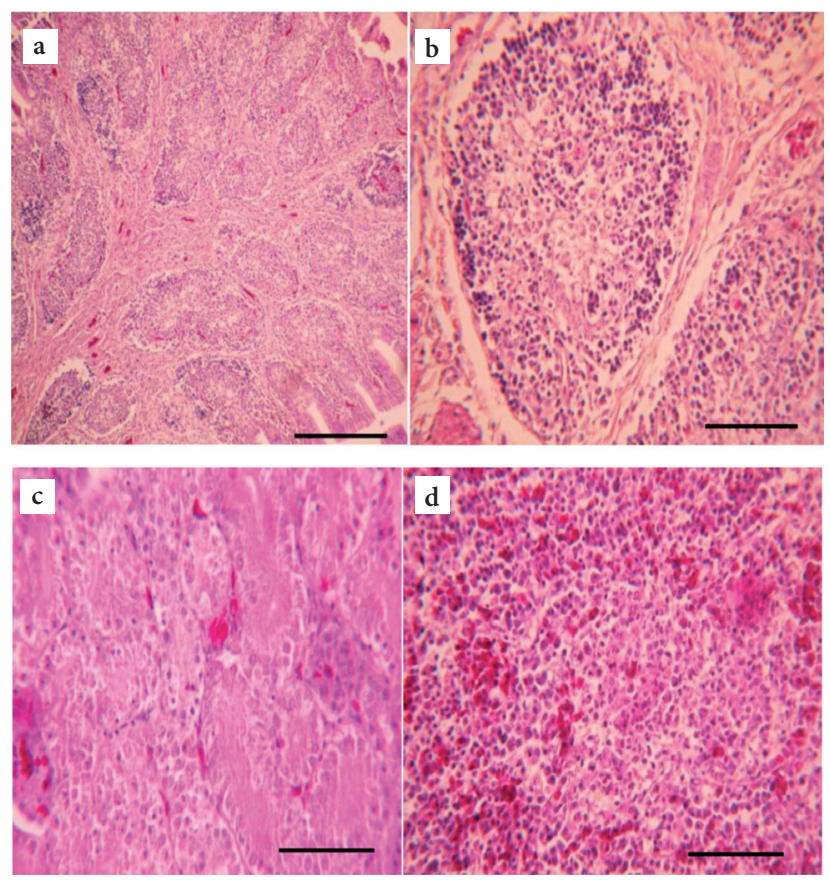

Fig. 1

Photomicrograph of IBD-affected organs

(a) Bursa of Fabricius. Degeneration and necrosis of lymphocytes in the bursal follicles and presence of cysts in the follicles with mild to moderate follicular atrophy (H\&E, bar: $150 \mu \mathrm{m})$. (b) Depleted lymphocytes were replaced by heterophils and debris cells (H\&E, bar: $40 \mu \mathrm{m})$. (c) Kidney. Tubular cell degeneration and tubular necrosis (H\&E, bar: $40 \mu \mathrm{m})$. (d) Spleen. Lymphocyte depletion in splenic lymphoid follicles and heterophils invasion (H\&E, bar: $40 \mu \mathrm{m})$.

I294, and S299, were present in the hvVP2 sequence of studied vvIBD isolates. The isolates lacked antigenically atypical vvIBDV characteristic amino acid residues, L324 and V321 (Eterradossi et al., 1998). The attenuated IBD isolates contained amino acid sequences concerned with propagation in cell culture (N279 and T284) (Brandt et al., 2001). Compared to D78 vaccine strain, hvVP2 sequences of the two attenuated isolates were similar, except for having a 253Q residue similar to vvIBD strains. No mutation at the major hydrophilic peak B, minor hydrophilic peak 1 , minor hydrophilic peak 2 , minor hydrophilic peak 3 and serine-rich heptapeptide motifs of the hvVP2 segment of the sequenced vvIBD isolates was seen, compared to the reference strain UK661. Interestingly, the mutation $254 \mathrm{G} \rightarrow \mathrm{S}$ was common among vvIBD isolates in this study, compared to UK661 and other Iranian vvIBD isolates.

\section{Phylogenetic analysis}

Using BLAST [http://blast.ncbi.nlm.nih.gov/Blast.cgi], the nucleotide sequences of Iranian isolates obtained in this study were evaluated to find other IBD isolates with highest identity. The phylogenetic relationship among IBD isolates with high nucleotide identity to the studied isolates and other well-known very virulent, classical, variant and attenuated IBD strains was determined using the Clustal W method (Fig. 2). Almost all of Iranian IBD isolates, including most of those sequenced in this study, fell into a distinct sublineage in the vvIBD lineage in the class of serotype 1, next to the reference isolate UK661. Some other Iranian isolates were classified beside the D78 vaccine strain, named as attenuated (vaccine) strains (Fig. 2). In the phylogenetic tree, Iranian vvIBD isolates are divided in two main subgroups. First subgroup consists of some isolates, mostly older ones, close to isolates from neighbor countries, like Iraq and Turkey (97.7-98.7\% of similarity). The second subgroup, in which mostly new Iranian isolates are placed, is more distant from neighbor countries' isolates 


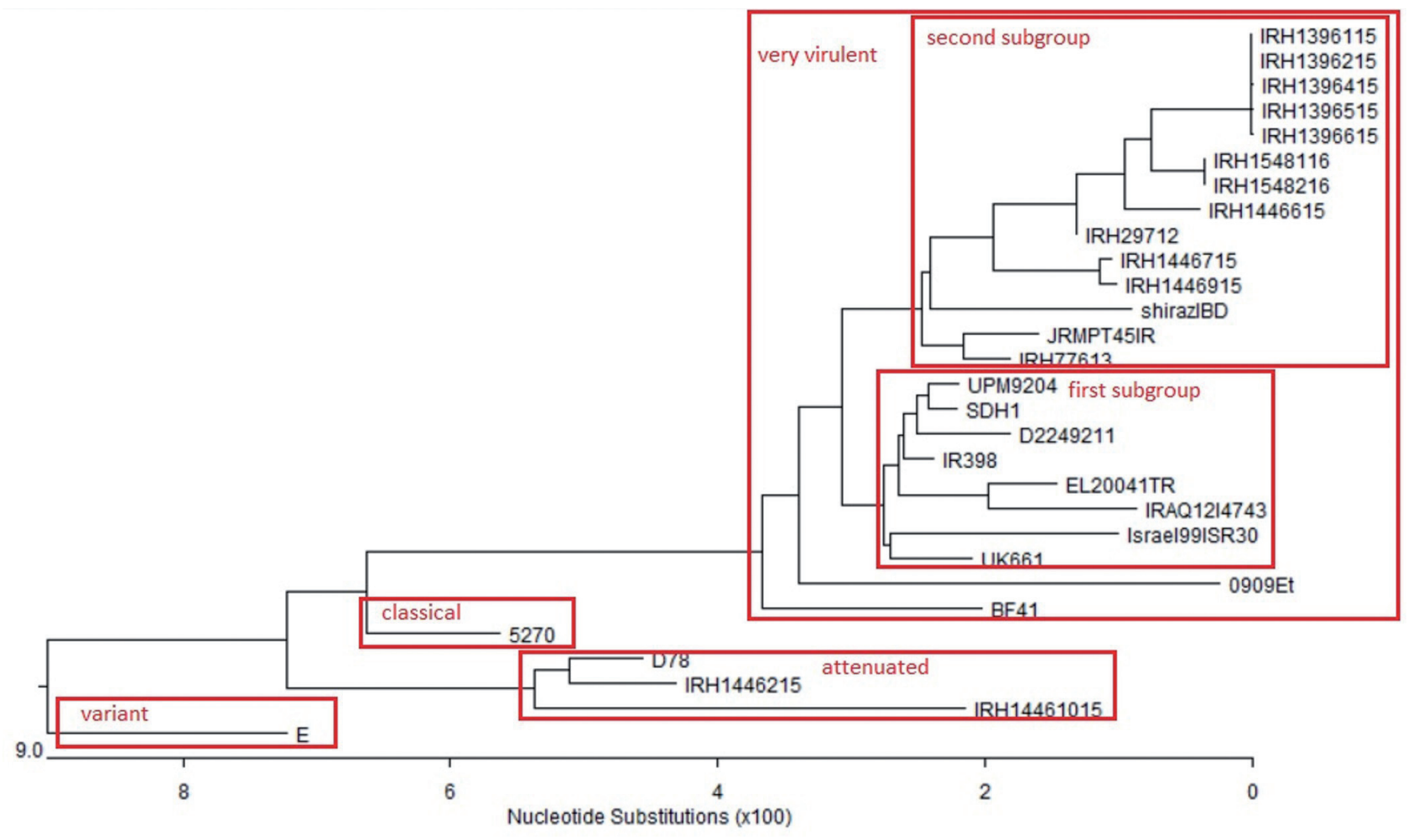

Fig. 2

Phylogenetic tree of the studied IBD isolates and other Iranian, reference and vaccine strains

The tree constructed by Clustal W method is based on the nucleotide sequence of hvVP2. Numbers at the nodes indicate the bootstrap confidence values calculated using 1000 boot strapping replicates. Acc. Nos. are shown in parenthesis.

(95.7-97.2\% of similarity). More classifications are seen in this subgroup. Surprisingly, Iranian vvIBD strains (SDH1, D2249211, Ghorashil and IR398) were closely related to a very virulent isolate (UPM9204) from Malaya (99-99.6\% of similarity). On the other hand, four Iranian isolates, including two of our sequenced isolates (IRH1446215 and IRH14461015) were clustered with the lineage of D78 vaccine strain.

\section{Discussion}

We gathered samples from IBD suspected outbreaks in broiler and layer farms from different regions of Iran during 2014-2015, thereby vvIBD strains circulates around the country. Typical macroscopical and histopathological lesions of the bursa of Fabricius were found similar to reports by other researchers (Hoque et al., 2001; Guvenc et al., 2004; Islam et al., 2008). Histopathological characteristics were concerned with a significant immunosuppression in affected flocks. However, mortality rates in the studied flocks are much lower than expected for vvIBD.
The sequence analysis revealed that ten isolates sequenced in this study had genetic characteristics related to vvIBD strains. vvIBD isolates from this study had a $G$ to $S$ mutation at position 254. The mutation was unique compared to other Iranian vvIBD isolates. Some studies on vvIBD strains in other countries have shown that mutations in hvVP2 region have been continued hereditarily to next generations and becoming stable in the populations (Xu, 2015). Hoque et al. (2001) reported that presence of 254 S mutation in a Malayan IBD isolate (94/273) was accompanied with lesser virulence (mortality rate $10 \%$ in SPF chickens). They suggested that the isolate should be assumed as a classical virulent IBD virus, in spite of possession of vvIBD characteristic amino acids. Actually, no one of broiler flocks, from which the bursal samples were collected, had mortality rates higher than $20 \%$. Currently, the only acceptable measure for identification of the vvIBD pathotype is mortality rate in SPF chickens (van Den Berg et al., 2004). Another notifiable mutation in one of the vvIBD isolates (IR/H1446.2/15) and the two attenuated strains (IR/H1446.2/15, IR/H1446.10/15) is $270 \mathrm{~A} \rightarrow \mathrm{T}$. This mutation has been found in the serotype 2 ( $\mathrm{OH}$ strain) and the $\mathrm{Cu} 1$ strains. On the other hand, the Malayan isolate 
(94/273) also had that amino acid substitution at position 270 from A to E. It is possible that both mutations are, at least partly, responsible for low mortality rate of vvIBD viruses circulating in Iranian broiler farms.

According to the phylogenetic analysis, Iranian vvIBD isolates are placed in a separate group, close to isolates from neighbor countries, although there are more classifications in that group. Most of recent Iranian vvIBD isolates, including the studied isolates, fell into an apart subgroup. The degree of genetic similarity of hvVP2 segment among the isolates sequenced in this study was relatively high (97.6-100\%), suggesting a common ancestor for all vvIBD viruses circulating in poultry farms of Iran today.

Considering the fact that studied isolates were gathered from different geographical regions of Iran, there was no relationship between geographical origin and genotype of the isolates. Genetic stability of the hvVP2 segment has been detected in Nigeria (Owoade et al., 2004) and Italy (Martin et al., 2007), because of low immune pressure or a static virus source (Owoade et al., 2004). However, when the range of comparison is prevailed, it is revealed that new Iranian isolates are partly different from older Iranian and neighbor countries' isolates (96.2-97.3\% similarity to Shiraz isolate and $95.7-96.7 \%$ to Iraq and Turkey isolates). The studied isolates also had the same degree of similarity to the reference vvIBD strain (UK661) and a Malayan strain (UPM9204) (96.7-96.9\%). Hence, it could be concluded that recent Iranian vvIBD strains are relatively different from the previous ones, necessitating a more detailed molecular and biological evaluation of these isolates.

Among sequenced IBD viruses, two were phylogenetically related to vaccine strains like D78 (genetic similarity 96-98.7\%). It would be logical to consider them as derivatives of D78 vaccine strain, while they have been isolated from broiler farms with gross pathological signs in the bursa. Threonine at position 284 and glutamine at position 253 are typical for vaccine strains (Brandt et al., 2001). It is documented that only a single point mutation at position 253 (N or Q) has created virulent variants of $\mathrm{D} 78$ vaccine strain (Brandt et al., 2001). The mentioned mutation (253Q) was seen in the two vaccine isolates. Such virulent variants of D78 vaccine strain, like GA-1, have been isolated from vaccinated flocks, particularly if the manufacturers' protocol has not been considered (Jackwood et al., 2008). The problem is quite common among poultry farmers in Iran. Therefore, it is of value to determine if the amino acid mutation in those two vaccine isolates is related with virulence reversion or not.

In conclusion, results of the present study revealed a widespread existence of very similar strains of vvIBD in poultry farms of Iran, differing to some extent from previous Iranian isolates available in GenBank. Particularly, they have new mutations in the hvVP2 region, which seems to become stable in Iranian vvIBD viruses. Some of those mutations might be associated with low mortality rates of vvIBD outbreaks in Iranian poultry flocks. More comprehensive studies on genetic and antigenic differences of new and previous isolates as well as probable modifications in their pathogenicity are needed to evaluate the significance of those changes.

Acknowledgements. We gratefully acknowledge the deputy of research office of Lorestan University for financial supporting.

\section{References}

Aghakhan SM, Fereidoni SR, Abshar N, Marunesi S, Sami Z (1996): Characterization of a highly virulent infectious bursal disease virus. Arch. Razi Institute 46/47, 55-63.

Amin OG, Jackwood DJ (2014): Identification and molecular analysis of infectious bursal disease in broiler farms in the Kurdistan Regional Government of Iraq. Trop. Anim. Health Product. 46, 1297-1301. https://doi.org/10.1007/ s11250-014-0643-0

Bayliss CD, Spies U, Shaw K, Peters RW, Papageorgiou A, Müller H, Boursnell ME (1990): A comparison of the sequences of segment A of four infectious bursal disease virus strains and identification of a variable region in VP2. J. Gen. Virol. 71, 1303-1312. https://doi.org/10.1099/00221317-71-6-1303

Becht H (1981): Infectious bursal disease virus. Cur. Top. Microbiol. Immunol. 90, 107-121. https://doi.org/10.1007/978-3$\underline{642-67717-5 \quad 5}$

Brandt M, Yao K, Liu M, Heckert RA, Vakharia VN (2001): Molecular determinants of virulence, cell trophism, and pathogenic phenotype of infectious bursal disease virus. J. Virol. 75, 11974-11982. https://doi.org/10.1128/ JVI.75.24.11974-11982.2001

Ceribaci AO, Bulut H, Gulacti I, Eruksuz Y, Bolat Y (2007): Presence of a Very Virulent Genotype of Infectious Bursal Disease Virus in Vaccinated Layer Hens in Turkey. Turkish J. Vet. Anim. Sci. 31, 105-111.

Cosgrove AS (1962): An apparently new disease of chickens. Avian Dis. 6, 385-389. https://doi.org/10.2307/1587909

Eterradossi N, Arnauld C, Toquin D, Rivallan G (1998): Critical amino acid changes in VP2 variable domain are associated with typical and atypical antigenicity in very virulent infectious bursal disease viruses. Arch. Virol. 143, 1627-1636. https://doi.org/10.1007/s007050050404

Guvenc T, Hazıroglu R, Yarım M, Tunca R (2004): Diagnosis of infectious bursal disease by immunoperoxidase technique. Vet. J. Ankara Univ. 51, 237-238.

Hoque MM, Omar AR, Chong LK, Hair-Bejoand Aini I (2001): Pathogenicity of Sspl-positive infectious bursal disease virus and molecular characterization of the hypervariable region. Avian Pathol. 30, 369-380. https://doi. org/10.1080/03079450120066377

Hosseini SD, Omar AR, Aini I (2004): Molecular characterization of an infectious bursal disease virus isolate from Iran. Acta Virol. 48, 79-83. 
Islam MN, Rashid SMH, Hoque MF, Juli MSB, Khatun M (2008): Pathogenicity of IBDV related to outbreaks in the vaccinated flocks and the causes of vaccination failure. J. Innov. Dev. Strategy 2, 22-30.

Jackwood DJ (2004): Recent trends in the molecular diagnosis of infectious bursal disease viruses. Anim. Health Res. Rev. 5, 313-316. https://doi.org/10.1079/AHR200490

Jackwood, D, Sommer S, Knoblich H (2001): Amino acid comparison of infectious bursal disease viruses placed in the same or different molecular groups by RT/PCR-RFLP. Avian Dis. 45, 330-339. https://doi.org/10.2307/1592972

Jackwood DJ, Sreedevi B, LeFever LJ, Sommer-Wagner SE (2008): Studies on naturally occurring infectious bursal disease viruses suggest that a single amino acid substitution at position 253 in VP2 increases pathogenicity. Virology 377, 110-116. https://doi.org/10.1016/j. virol.2008.04.018

Jenberie S, Lynch SE, Kebede F, Christley RM, Gelaye E, Negussie H, Asmare K, Ayeleta G (2014): Genetic characterization of infectious bursal disease virus isolates in Ethiopia. Acta Tropica 130, 39-43. https://doi.org/10.1016/j. actatropica.2013.09.025

Lasher HN, Shane SM (1994): Infectious bursal disease. World Poultry Sci. J. 50, 133-166. https://doi.org/10.1079/WPS19940013

Le Nouën C, Rivallan G, Toquin D, Darlu P, MorinY, BevenV, de Boisseson C, Cazaban C, Comte S, Gardin Y, Eterradossi $\mathrm{N}$ (2006): Very virulent infectious bursal disease virus: reduced pathogenicity in a rare natural segment-Breassorted isolate. J. Gen. Virol. 87, 209-216. https://doi. org/10.1099/vir.0.81184-0

Lin Z, Kato A, Otaki Y, Nakamura T, Sasmaz E, Ueda S (1993). Sequence comparisons of a highly virulent infectious bursal disease virus prevalent in Japan. Avian Dis. 37: 315-323. https://doi.org/10.2307/1591655

Martin AM, Fallacara F, Barbieri I, Tosi G, Rivallan G, Eterradossi N, Ceruti R, Cordioli P (2007): Genetic and antigenic characterization of infectious bursal disease viruses iso- lated in Italy during the period 2002-2005. Avian Dis. 51, 863-872. https://doi.org/10.1637/7904-020107-REGR.1 Mohamed MA, Elzanaty KES, Bakhit BM, Safwat MM (2014): Genetic Characterization of Infectious Bursal Disease Viruses Associated with Gumboro Outbreaks in Commercial Broilers from Asyut Province, Egypt. Int. Scholarly Res. Notices Vet. Sci., Article ID 916412, 9 pages.

Owoade AA, Mulders MN, Kohnen J, Ammerlaan W, Muller CP (2004): High sequence diversity in infectious bursal disease virus serotype 1 in poultry and turkey suggests WestAfrican origin of very virulent strains. Arch. Virol. 149, 653-672. https://doi.org/10.1007/s00705-003-0270-y

Snyder DB, Lana DP, Savage PK, Yancey FS, Mengel SA, Marquardt WW (1988): Differentiation of infectious bursal disease viruses directly from infected tissues with neutralizing monoclonal antibodies: evidence of a major antigenic shift in recent field isolates. Avian Dis. 32, 535-539. https:// doi.org/10.2307/1590924

Vakharia VN, He J, Ahmed B, Synder DB (1994): Molecular basis of antigenic variation in IBDV. Virus Res. 31, 265-273. https://doi.org/10.1016/0168-1702(94)90009-4

van den Berg T, Gonze M, Meulemans G (1991): Acute infectious bursal disease in poultry: isolation and characterisation of a highly virulent strain. Avian Pathol. 20, 133-143. https://doi.org/10.1080/03079459108418748

van den Berg TP, Morales D, Eterradossi N, Rivallan G, Toquin D, Raue R, Zierenberg K, Zhang MF, Zhu YP, Wang CQ, Zheng HJ, Wang X, Chen GC, Lim BL, Muller H (2004): Assessment of genetic, antigenic and pathotypic criteria for the characterization of IBDV strains. Avian Pathol. 33, 470-476. https://doi. org/10.1080/03079450400003650

Xu MY, Lin S-Y, Zhao Y, Jin J, Tang N, Zhang GZ (2015): Characteristics of very virulent infectious bursal disease viruses isolated from Chinese broiler chickens (2012-2013). Acta Tropica 141, 128-134. https://doi.org/10.1016/j. actatropica.2014.10.003 